\title{
LA PROBLEMÁTICA DE LA ORFANDAD EN EL CHILE DEL SIGLO XIX: UNA MIRADA AL ROL DEL ESTADO
}

\author{
David Home Valenzuela \\ Universidad Católica Silva Henríquez, Chile \\ dhome@ucsh.cl
}

\begin{abstract}
RESUMEN
En este artículo se estudia el papel del Estado en la atención de la orfandad en Chile durante el siglo XIX. A través de una visión panorámica, el texto pone su atención en actores y momentos claves analizando el desarrollo y la evolución de la beneficencia pública. Dada la impronta liberal con que el Estado chileno se construyó tras la Independencia y su persistente falta de recursos económicos, la problemática social de la orfandad fue asumida principalmente por la sociedad civil y la Iglesia Católica. Hacia el final del siglo, y gozando de una institucionalidad más sólida, el Estado chileno comenzó a tener un rol más protagónico que se consolidaría en la siguiente centuria.
\end{abstract}

Palabras clave: Política social, Formación del Estado, Huérfanos, Chile. 


\title{
THE ISSUE OF ORPHANHOOD IN CHILE DURING NINETEENTH CENTURY: A LOOK TO THE ROLE OF THE STATE
}

\begin{abstract}
This article studies the role played by the Chilean state in the care of orphans during the nineteenth century. Through an overview of the whole century, the work focuses on both actors and key moments to analyze the evolution of the public beneficence. Given the liberal character with which the Chilean State was built after the Independence and the persistent lack of economic resources, the social issue of the orphanhood was mainly assumed by the civil society and the Catholic Church. Towards the end of the century, and enjoying a stronger institutionality, the Chilean State began to play a more relevant role that would be deepened in the following century.
\end{abstract}

Keywords: Social policy, State formation, Orphans, Chile. 


\section{INTRODUCCIÓN}

El problema de la orfandad ha sido reconocido por numerosos estudiosos como uno de las principales males sociales del Chile postcolonial, llegando incluso a ser considerado, por su alcance y magnitud, casi como un sello identitario nacional ${ }^{1}$. En este artículo se revisa y analiza de forma panorámica la labor desarrollada por el Estado chileno durante el siglo XIX en esta materia. A lo largo del período estudiado se constata que ante el limitado interés con que sus autoridades políticas abordaron estas problemáticas durante gran parte del siglo, la tarea fue asumida por la sociedad civil y la Iglesia Católica, actores que jugaron un importante papel en las materias vinculadas con la beneficencia pública, en general, y en la atención y el cuidado de los huérfanos, en particular. En este periodo es posible apreciar que tras un primer momento marcado por la colaboración y complementariedad entre estos distintos actores se pasará a una etapa caracterizada por una mayor injerencia estatal. En este proceso influirán tanto la creciente secularización que experimentó la sociedad chilena hacia fines del siglo XIX como al fortalecimiento del Estado con posterioridad a la Guerra del Pacífico.

\section{ANTECEDENTES COLONIALES PARA UN PROBLEMA NACIONAL: LA FUNDACIÓN DE LA CASA DE HUÉRFANOS DE SANTIAGO}

En 1758 comenzaba su trayectoria institucional la Casa de Huérfanos de Santiago, gestada gracias a un legado de un particular, el comerciante valenciano Pedro Tisbe, quién al fallecer dejó parte de su fortuna para ser utilizada en alguna obra de beneficencia. Otro connotado vecino de Santiago, Juan Nicolás de Aguirre, conocido también como el Marqués de Montepío, quedó a cargo del cumplimiento de la voluntad de Tisbe y, siguiendo su mandato, optó por destinar dichos recursos a la fundación de una casa de huérfanos. En su presentación ante la Real Audiencia de Santiago, en la que solicitaba el permiso oficial para la instalación de dicha institución, Aguirre planteó que un establecimiento de ese tipo serviría para palear el problema social de los "muchos hijos expósitos, que se malogran por este defecto". En su planteamiento señaló que eran numerosos los nińos "verdaderamente pobres" que recorrían la ciudad "de casa en casa" solicitando alimentos, y sin la debida protección y cuidado que por su edad requerían (Vicuña Mackenna 1974). La fundación de un hogar para acoger a los huérfanos, además, permitiría la entrega de valores como la

1 Son numerosos los textos que se refieren a este punto, tan sólo a modo de referencia podemos mencionar los trabajos de Gabriel Salazar (1990) y Sonia Montecino (1991). 
fe cristiana y el trabajo, por lo que a todas luces una fundación de una institución de este tipo sólo entregaría beneficios a la sociedad.

La petición de Aguirre fue favorablemente acogida por las autoridades coloniales aunque su implementación definitiva tomó mucho más tiempo que el presupuestado. La Real Audiencia de Santiago autorizó su funcionamiento en 1759 con una capacidad inicial de cincuenta menores y, años más tarde, en 1771, la Corona Española le concedió por Real Cédula una renta para colaborar con el mantenimiento de los menores. Sin embargo, a pesar de este apoyo inicial, recién en 1780 la institución recibiría la aprobación real definitiva que sancionaría su instalación.

Si bien se tiende a asimilar el concepto de huérfano con la ausencia de padre y madre a causa de su fallecimiento, en el periodo colonial y durante el siglo XIX dicho concepto más bien se utilizó para señalar a aquellos menores que eran abandonados por sus progenitores. Del mismo modo, como sinónimo de huérfano se empleaba el término de expósito, debido a que la labor de la Casa de Huérfanos comenzaba cuando un menor abandonado era expuesto en las proximidades de la Casa o bien en el torno de ella, una instalación especialmente diseńada para facilitar el depósito de menores y que garantizaba el anonimato de quien realizaba dicho acto. Una vez ingresado en la institución, el huérfano debía cumplir con distintos procesos, asociados a su edad. Si el menor era un lactante, era inmediatamente entregado a una ama de pecho, contratada por la institución y que habitaba en ella: su labor consistía en amamantarlo hasta que el menor dejara esa etapa. Luego el menor era entregado a una segunda mujer, conocida como seca, que en este caso vivía fuera del recinto. Ella se encargaba del cuidado y mantención del menor, gracias a un sueldo pagado por la Casa de Huérfanos, hasta la edad de cuatro o seis años de edad. Tras esa etapa el menor volvía a la Casa para recibir nociones elementales de enseñanza y también los preceptos cristianos básicos. Algunos de ellos eran instruidos para que solicitasen limosnas en la vía pública, las que eran utilizadas para solventar los gastos de la institución.

El funcionamiento de la Casa de Huérfanos de Santiago se vio afectado por frecuentes apuros económicos. Con ingresos acotados, la disposición de recursos fue siempre limitada y las necesidades crecientes. Los egresos incluían, además de los gastos de habilitación y mantención del edificio, los montos que debían disponerse para la alimentación y vestuario de los menores, así como también para el pago de las mujeres que se hacían cargo de su cuidado. El número de menores rápidamente superó las cifras proyectadas inicialmente: sólo entre 1772 y 1776 ingresaron a la institución 127 infantes. Si bien se hacían esfuerzos por brindarles las mejores condiciones posibles casi el sesenta por ciento del total de niños 
ingresados entre 1797 y 1818 falleció a causas de diversos males (Delgado 1984).

A pesar de los esfuerzos de sus administradores, la Casa de Huérfanos de Santiago no logró constituirse en una solución eficaz para el problema de la orfandad debido a su limitado alcance y a la persistente escasez de recursos.

\section{LAS PRIMERAS DÉCADAS DE LA REPÚBLICA Y LA CREACIÓN DE LA JUNTA DE BENEFICENCIA DE SANTIAGO}

Tras la Independencia las principales preocupaciones de las autoridades chilenas estuvieron centradas en garantizar la autonomía del país respecto a la Corona española y construir instituciones que administraran los recursos económicos necesarios con que se contaba con el fin último de asegurar la soberanía territorial. Los requerimientos vinculados con la beneficencia pública, en general, y el cuidado y atención de los huérfanos, en particular, no eran considerados como prioritarios.

A pesar de ello, durante las primeras décadas del siglo XIX, la Casa de Huérfanos de Santiago siguió funcionando, aunque caracterizada por una acuciante inestabilidad, lo que se refleja, por ejemplo, en su cierre de manera temporal en 1821 y su reapertura al año siguiente, por orden de Bernardo O'Higgins. Ante esta precaria situación, muchos menores eran reubicados en otras ciudades del país o bien entregados a particulares para su cuidado y, en algunos casos, para el desempeño de actividades domésticas (Delgado 1984).

Algunos años más tarde, y al tiempo que el Estado lentamente se iba consolidando, el esfuerzo de las autoridades políticas tomaría forma en la creación de una institucionalidad que se hiciera cargo de esta materias centralizando los esfuerzos en materia de beneficencia pública que hasta entonces se desplegaban de manera desorganizada. Un primer paso en tal sentido lo constituyó la creación en 1832 de la Junta Central de Beneficencia y Salud Pública, que al estar encargada de "velar sobre todos los establecimientos de beneficencia y salud pública de la capital y provincias", tomó a su cargo la Casa de Huérfanos de Santiago y nombró como miembros de su comisión administrativa a Pedro Urriola, Manuel Carvallo y Antonio Vial (Delgado 1984).

Si bien la organización de esta nueva institucionalidad representó un avance, su creación no significó que los problemas de la beneficencia pública pudiesen ser considerados como parte del pasado. Al poco tiempo de instalada, y a petición de la Asamblea Provincial de Santiago, la Junta Central de Beneficencia y Salud Pública fue reemplazada por la Junta 
Directora de Hospitales y Casa de Expósitos de Santiago, que designó a Cipriano Pérez como administrador de la Casa de Huérfanos. Con el paso de los años, esta nueva repartición sería denominada indistintamente Junta Directora de los Establecimientos de Beneficencia o Junta de Beneficencia (Laval 1949: 43-47).

En su composición interna las juntas funcionaron como corporaciones de carácter mixto, público y privado, toda vez que dependían el Ministerio del Interior para la elección de sus integrantes, administraban recursos fiscales y también donativos particulares, aunque en la práctica no formaban parte del aparato estatal (Ponce de León 2011). Al igual que en otras instancias organizadas que prestaban colaboración al Estado, los miembros de las juntas eran personas destacadas, miembros de la elite o cercanos a ella, que voluntariamente prestaban colaboración en las materias vinculadas con la beneficencia y que, por su labor, no recibían remuneración. Siguiendo lo planteado por Macarena Ponce de León, es posible señalar que, en muchos sentidos, las juntas de beneficencia se asemejaban a las asociaciones propias del Antiguo Régimen, en las que predominaba una jerarquía determinada, de participación voluntaria, selectivas en su composición social, autónomas internamente pero dependientes de la autoridad central (Ponce de León 2011).

Un estudio prosopográfico de los miembros de las juntas de beneficencia entre 1822 y 1886 da cuenta de la participación de 194 individuos en estas corporaciones. Se trata de una actividad eminentemente masculina, desarrollada por hombres pertenecientes al mismo grupo social, activos políticamente y estrechamente vinculados familiarmente. En las nóminas aparecen numerosos ministros de Estado, parlamentarios, ministros de justicia, profesores universitarios, todas ellas, funciones de alta responsabilidad política y social. En cuanto a la formación profesional, en su gran mayoría eran abogados, religiosos, militares y médicos. La presencia de religiosos, eso sí, iría disminuyendo a través del siglo XIX al mismo tiempo que los enfrentamientos entre el Estado liberal y la Iglesia Católica fueron adquiriendo mayor resonancia.

A contar de la década de 1830, la Junta de Beneficencia de Santiago tuvo a su cargo la administración de los hospitales San Francisco de Borja y San Juan de Dios, que hasta la década anterior habían sido administrados por religiosos y que habían sido fundados en el periodo colonial, a los que se sumaba la Casa de Expósitos. Décadas más tarde, la Junta ya controlaba, también, el Hospicio de Pobres de ambos sexos, el Cementerio General y la Casa de Orates con lo cual su rol como agente centralizador de la beneficencia pública se fue consolidando poco a poco, en un desarrollo que seguiría profundizándose a lo largo del siglo. 
En materia de funcionamiento, la Junta de Beneficencia tenía por objetivo principal controlar la organización interna y la administración económica de las instituciones bajo su tutela. Autorizaba la vigencia de sus reglamentos internos y cautelaba la inversión de los fondos de que ellos disponían. Estos últimos provenían principalmente de las subvenciones estatales y municipales, aunque los legados de particulares eran también muy relevantes. Estos últimos no consistían solamente en recursos económicos en efectivo, sino que también incluían propiedades y bienes inmuebles cuyos productos, derivados de arriendos y enajenaciones, iban en beneficio de las instituciones a las que fueron entregados.

En un plano comparativo, los hospitales y la Casa de Huérfanos fueron las instituciones que recibieron mayor atención estatal en términos financieros, lo que se sumaba a las limosnas y donaciones particulares que auxiliaban su funcionamiento. En este contexto, y a pesar de tales contribuciones, es posible afirmar que el Estado se limitó a cumplir un rol meramente subsidiario, pues si bien no asumió de forma directa la administración de estas instituciones, sí colaboró de forma constante con subvenciones. La subvención representó, por lo tanto, el principal mecanismo a través del cual el Estado se hizo presente en la beneficencia pública, toda vez que dentro de la impronta liberal se consideraba que dichos requerimientos y sus necesidades asociadas eran excesivas y ajenas a sus facultades (Salinas 1983).

\section{LA PARTICIPACIÓN DE LA SOCIEDAD CIVIL: ASOCIACIONES Y SOCIEDADES DE BENEFICENCIA}

Tanto por las limitaciones y desinterés del Estado para atender las necesidades de la beneficencia pública como por su propia iniciativa y voluntad, otros actores sociales decidieron organizarse con el propósito de buscar soluciones que complementaran los esfuerzos que se emprendían desde la institucionalidad. Conforme transcurrió el siglo XIX la sociedad civil acentuará un proceso de organización marcado por la adopción de formas de sociabilidad modernas, impregnadas de un espíritu ilustrado que buscaba además de la genuina preocupación por la condición vital del otro, su moralización y regeneración en función de los intereses y del modelo de sociedad que las clases dirigentes buscaban construir.

Un primer antecedente en este sentido lo constituyó la llamada Sociedad de Amigos del País, cuyo funcionamiento fue aprobado en 1813 por el Cabildo de Santiago y que tenía como finalidad "promover el adelanto del país en todos los ramos de la industria", poniendo especial énfasis en fomentar la enseñanza de la agricultura, impulsar la educación de mujeres, mejorar la suerte de los presos y realizar otras obras de caridad en general. 
Pertenecieron a esta sociedad, entre otros, Juan Egaña, Mateo Höevel, Domingo Eyzaguirre, José Miguel Infante y Manuel de Salas. Este último, hombre muy activo en los temas relacionados con la beneficencia, dirigió también la llamada Casa de Corrección y Enseñanza que, refundada en 1824, tenía como objetivo alejar a las prostitutas de su oficio brindándoles educación y normas moralizadoras, a la vez que instruyéndolas en la realización de trabajos industriales (Cárdenas 1991).

La labor desarrollada por la Sociedad de Amigos del País se vio truncada por la Reconquista teniendo que pasar varios ańos para que nuevas asociaciones emergieran de la sociedad civil y se consolidaran en el tiempo. A mediados de la década siguiente, por ejemplo, se intentó organizar la llamada Sociedad Filantrópica de Amantes de la Patria, pero el esfuerzo sería vano al no alcanzar proyección en su tarea (Saldivia y De la Jara 2001).

Hacia fines de la década de 1830 los esfuerzos de algunos particulares comenzaron a cristalizarse en acciones concretas y de mayor pervivencia en el tiempo. A instancias de José Miguel de la Barra, activo colaborador en materias de beneficencia, se creó la Sociedad Nacional de Agricultura y Beneficencia en 1838, que además tuvo como miembros fundadores a Andrés Bello, Manuel de Salas, Pedro Palazuelos, entre otros. La Sociedad se organizó con el propósito de difundir y destacar la importancia de la agricultura, no tan sólo en términos económicos sino que también en función de su impacto social. Dentro de esta última esfera, la Sociedad puso especial atención en la situación y condición de los sectores más desfavorecidos de Chile; a partir de su iniciativa se comenzarán a implementar medidas que buscaban morigerar los vicios, inculcar medidas de salubridad e higiene y también generar instancias de educación popular.

José Miguel de la Barra se transformó en uno de los hombres públicos más activos en materia de beneficencia durante estos años. Además de participar en la Sociedad Nacional de Agricultura y Beneficencia, fue miembro de la Sociedad Cristiana para el Socorro de los Pobres Vergonzantes y desde su cargo de Intendente de Santiago colaboró de manera trascendental en el establecimiento del Asilo del Salvador, institución que tuvo por propósito albergar a mujeres viudas, huérfanas o que hubiesen quedado en situación de pobreza o abandono. Se buscaba rescatar a aquellas que "por su pobreza necesariamente habrían quedado sin educación, y por consiguiente de poco o de ningún provecho podían ser para la sociedad" (La Revista Católica $1 / 11 / 1844)$. Al igual que en otros recintos de este tipo destinados para población femenina, se dispuso que en su interior, aparte de habitación y educación gratuita, funcionasen algunas maquinarias para que las internas aprendieran las técnicas del hilado y las faenas de la costura (Cárdenas 1991: 60). Para remediar la situación de los huérfanos, José Miguel de la 
Barra sugería que se organizasen sociedades de señoras "que atendiesen a la crianza, educación y establecimiento de los niños expósitos, en calidad de madres adoptivas" (Durán 1851: 18).

La solución planteada por De la Barra, esto es, la organización de sociedades que colaborasen en el auxilio de los huérfanos, fue finalmente el camino que siguió la sociedad civil chilena, especialmente a partir de la década de 1840 . La toma de conciencia respecto a este problema social y el análisis de las causas que lo motivaban fueron a la par con el surgimiento de soluciones para abordarlo. El objetivo final de estas iniciativas buscaba el mejoramiento de las condiciones de vida de estos menores, para lo cual se propuso tanto el fortalecimiento de las instituciones de acogida que ya existían como la creación de otras nuevas que los albergaran y cuidaran conforme a los valores culturales del periodo.

Las voces que clamaban por una mayor atención respecto al problema de los huérfanos no sólo procedían de Santiago. En 1843, por ejemplo, un regidor de Valparaíso planteó en el seno de la corporación municipal la necesidad de establecer una casa de expósitos en esa ciudad. Justificó su solicitud señalando que en repetidas ocasiones se escuchaba decir que en las distintas quebradas que rodean la ciudad aparecían "miembros despedazados de niños que han sido arrojados a ellas por el crimen o la miseria de sus padres, que no tienen como alimentarlos" (Pinto y Salazar 2002: 53). En La Serena, hacia fines de esa misma década, un miembro de la Sociedad de Beneficencia local presentó una solicitud de similar tenor. En su presentación, Marcelo Riveros abogó por la instalación de una casa de huérfanos que acogiese a los numerosos menores abandonados existentes en la zona. Si bien su propuesta establecía una categorización bastante estricta respecto a los niños que ella debía acoger, al asociar su origen social y la causa de su abandono con el rol futuro que debía jugar en la sociedad, ella respondía a una necesidad imperiosa que el solicitante dejaba en claro en su planteamiento (Riveros 1849).

Tal como vemos, en las sociedades de beneficencia numerosos particulares de espíritu filantrópico e ilustrado expresaron su preocupación por este tipo de problemas y propusieron alternativas para colaborar con la infancia abandonada. Su alcance como solución definitiva a este problema fue, por cierto, modesto, siendo dependiente del empuje de sus impulsores y de la solvencia económica que las instituciones que se creaban lograban alcanzar. Para formar una sociedad de beneficencia bastaba la voluntad de un grupo de personas que, convocadas por un interés social, se dieran a la tarea de darse una organización y obtener el financiamiento para las actividades o los fines para los cuales se habían reunido. Además de las sociedades de beneficencia organizadas por mujeres y de aquellas 
vinculadas directamente con la Iglesia Católica, fueron también relevantes las establecidas por colonias extranjeras que, además de servir como punto de encuentro y reunión de foráneos con residencia permanente o de paso por Chile, colaboraron de manera bastante activa y consistente en el tiempo con asilos y casa de huérfanos.

Todas estas organizaciones buscaban cooperar con la sociedad en una tarea que el Estado desarrollaba de manera limitada a través de una institucionalidad en construcción. A la Junta de Beneficencia de Santiago, organizada en 1832, se sumarían en las décadas siguientes la Junta Directora de Establecimientos de Beneficencia de Valparaíso, establecida en noviembre de 1845; la de Talca, creada en julio de 1846; la de Concepción, regulada en noviembre de 1846; la de Copiapó, que comenzó a operar en mayo de 1850; la de Vallenar, en funcionamiento desde agosto de 1853; la de San Felipe, instalada en julio de 1857; y las de Los Andes y Chillán, ambas en conformación desde octubre de ese mismo ańo. Para cada caso, el Gobierno dispuso reglamentos y ordenanzas particulares donde se replicaba el modelo ya descrito para la de Santiago (Laval 1949: 46).

\section{EL APORTE DE LA IGLESIA CATÓLICA}

Con un Estado escasamente presente, le correspondió a la Iglesia Católica, ya sea por iniciativa de su jerarquía o bien por el impulso de algunos de sus miembros más activos, la tarea de encauzar los esfuerzos en materias de beneficencia y, más específicamente, en el auxilio de los huérfanos. Se ha calculado que al menos once de las trece instituciones fundadas entre 1844 y 1895 para atender a huérfanos o a menores abandonados estaban en directa relación con la Iglesia, ya sea en cuanto a su establecimiento, o bien respecto a su administración, lo que da debida cuenta de la relevancia que tuvo la movilización y organización católica en estas materias (Milanich 2009).

Fueron muy importantes durante este periodo las obras dirigidas por el presbítero Blas Cañas. Este notable benefactor, inspirado en las deplorables condiciones de vida de la infancia abandonada, concibió la fundación de la llamada Casa de María, contando para ello con la importante colaboración de las señoras de la Congregación del Salvador. La Casa de María, tenía como objetivo brindar "asilo para las niñas sin recursos ni protección", al mismo tiempo que "socorrer las necesidades espirituales y corporales de las jóvenes desvalidas" (Román 1887: 4). Fundada en 1856 y con una matrícula inicial de 24 menores, pronto fue necesaria la adquisición de un local más amplio con el objeto de atender las crecientes necesidades que iban surgiendo. Esta idea concitó el apoyo de gran parte de la comunidad 
de Santiago, gracias a lo cual fue posible reinaugurarla el 3 de enero de 1859

En la Casa de María se recibían huérfanas pobres de "familia decente" con una edad no inferior a tres años. Las de procedencia más humilde eran educadas para ser sirvientas eficientes "que conociendo los deberes religiosos y los quehaceres de su oficio", sabrían dar gusto en cualquier hogar cristiano. Las niñas provenientes de "familias decentes", divididas en cuatro secciones de acuerdo a su edad, recibían instrucción religiosa y práctica, centrada en enseñar las primeras letras y las labores domésticas, tales como lavado y planchado de ropa, "cocinería y dulcería, nacional y extranjera", en el entendido de que la entrega de cualquier otro tipo de conocimientos "lejos de aprovechar a niñas como las que ella asila, las tornarían orgullosas y presumidas" (Román 1887: 4).

En agosto de 1872, el presbítero Blas Cañas instaló una casa en la calle de Santa Rosa, el Patrocinio San José, institución que tenía por objetivo acoger a niños huérfanos, abandonados o carentes de recursos. Especial atención le brindó a aquellos hijos de personas que "después de haber tenido fortuna la han perdido, [...] que esos son en realidad los más desgraciados y los que a mayores peligros están expuestos" (Román 1886: 308). Hasta el año 1886, el Patrocinio San José había asilado cerca de cuatrocientos niños, los que fiel al modelo predominante en este tipo de instituciones ligadas a la Iglesia Católica, recibían una educación con fuerte énfasis en la enseñanza práctica, con talleres de carpintería, sastrería, zapatería, encuadernación de libros, reparación de pianos y relojería.

Tal como hemos reseńado, el problema de la orfandad y de la infancia abandonada no se limitaba tan sólo a Santiago. En provincias la situación de los huérfanos preocupaba de igual forma a importantes sectores de la sociedad, por lo que, replicando el modelo puesto en funcionamiento en la capital, también se crearían instituciones que velarían por la educación y el asilo de aquellos menores desprotegidos. En el caso de Valparaíso, fue muy relevante el Asilo del Salvador de Valparaíso, fundado en 1857 por un grupo de señoras de la alta sociedad porteńa, con el objetivo de atender a niñas huérfanas o de escasos recursos. Acogió en su primer año a 38 internas, ampliando su matrícula hasta llegar a 60 un año después (Lamarca,1859: 5). En su interior, como parte de la formación general se impartían cursos de religión, lectura, escritura y aritmética elemental; asimismo, y para propiciar la inserción social de las internas, se les dictaban cursos de costura, zapatería, bordado, lavado y planchado de ropa. En lo administrativo, la dirección y gestión financiera externa orientada a captar aportes, corría por cuenta de las socias fundadoras, mientras que el régimen interno del Asilo quedó en un comienzo a cargo de Hermanas 
de la Providencia, quienes durante esos mismos años colaboraban también en la administración de la Casa de Huérfanos de Santiago, y luego pasó a manos de las Hermanas de la Caridad, otra congregación muy activa en estas materias.

Si el origen de la mayoría de las instituciones que hemos reseñado tenía como denominador común la constatación de la desmedrada situación de los huérfanos, enmarcado en el escaso interés con que el Estado abordaba el problema social de la infancia abandonada, el surgimiento del Asilo de la Purísima no escaparía a esta norma, aunque sí poseería una característica peculiar. Su creación estuvo estrechamente vinculada con un suceso puntual: las graves inundaciones provocadas por el desborde del río Mapocho, debido a los temporales de viento y lluvia que afectaron a la zona central de Chile durante el invierno de 1877. Por tal razón, y a instancias de "piadosas señoras y respetables caballeros", el Arzobispo de Santiago, Rafael Valentín Valdivieso, decidió fundar una casa que le diera asilo y educación a los muchos niños que quedaron "desnudos y hambrientos" después del trastorno climático (Gatica 1884: 134-135).

Tal como hemos visto, en la mayoría de los casos reseńados predominó el modelo de Asilo, concebido como una institución en la cual su administración estaba a cargo de miembros de la Iglesia Católica, ya fueran religiosos individuales o bien congregaciones religiosas, y que contaba con una red de colaboradores que ayudaban al sostenimiento financiero de la obra. En su interior, además de brindar alojamiento y comida a los menores, se les entregaba instrucción en primeras letras y también de índole práctica que les permitiera ejercer un oficio una vez que salieran del recinto.

\section{FIN DE SIGLO: UN ESTADO MÁS ACTIVO Y REALIZADOR}

El panorama general que hemos reseñado sufrirá importantes cambios en las últimas décadas del siglo XIX. El creciente conflicto entre el Estado liberal y la Iglesia Católica ejercerá un importante impacto en la beneficencia, disputa que se verá reflejada en la situación de una institución específica, el Asilo de la Patria. Al mismo tiempo, tras la Guerra del Pacífico, el Estado dispondrá de mayores recursos que, sumados a la voluntad gubernamental, le permitirá emprender nuevas tareas en materia de beneficencia pública asumiendo responsabilidades que antes habían sido tomadas por otros actores sociales (Sunkel 2011).

La Guerra del Pacífico es un hito trascendental en la historia del Chile postcolonial. El conflicto bélico que enfrentó a Chile con Perú y Bolivia no sólo terminó modificando las fronteras de estos países y determinando con ello la incorporación de vastos territorios ricos en salitre a soberanía 
chilena, sino que también trajo consigo importantes consecuencias sociales derivadas de los enfrentamientos militares. El problema de la orfandad surgida por la muerte de soldados chilenos en combate fue una de ellas.

Tras el inicio de la Guerra del Pacífico la sociedad chilena se movilizó ampliamente y demostró con ello un profundo sentido de unidad nacional. A las manifestaciones de patriotismo propias de un conflicto bélico, se sumó una activa cooperación hacia los canales que se dispusieron para enfrentar los múltiples desafíos generados por la contienda.

Junto con la natural preocupación por el desarrollo de la guerra, aparecieron en la opinión pública voces que llamaron la atención respecto a otros temas derivados directamente de las consecuencias de ella, tales como el cuidado y la atención de los heridos y el auxilio de las familias de aquellos que partían a los campos de batalla. Si bien la preocupante condición en que quedaban los huérfanos derivados de la guerra se hizo evidente ya desde el inicio del conflicto bélico, este problema social alcanzó mayor notoriedad tras el Combate Naval de Iquique. El heroico desarrollo y desenlace de este encuentro marítimo conmovió profundamente a la sociedad chilena (Home 2007)

Surgido de la iniciativa del presbítero Ramón Ángel Jara, se fundó el 20 de julio de 1879 el Asilo de la Patria de Nuestra Señora del Carmen, institución en la que se brindaría alojamiento, alimentación e instrucción a los hijos de los soldados que fallecieran en la guerra, tanto en los campos de batalla como a consecuencia de heridas o enfermedades adquiridas durante la campaña.

Para admitir a un niño en el Asilo no era necesario que fuera huérfano de madre, pues se aceptaba también a aquellos menores que hubiesen perdido como, consecuencia de la guerra, a quien cumpliese el rol paterno, fuese un hermano, tío, abuelo o protector. Los asilados debían tener entre 6 y 14 años de edad. En el interior recibirían hospedaje, alimentación y educación católica, siendo divididos en dos secciones completamente separadas, una para los hijos de oficiales y otra para los descendientes de soldados y clases.

La institución gozó de un importante respaldo por parte de la sociedad chilena durante sus primeros años de vida, preocupada de solventar las necesidades de los que eran llamados los "huérfanos más gloriosos de la patria”. Sin embargo, tras el fin de las actividades bélicas de mayor importancia, en 1881, la institución comenzó a ser objeto de numerosas críticas por parte de los sectores liberales que, en síntesis, cuestionaban el marcado carácter eclesiástico que, a su juicio, había adquirido el Asilo. 
Esta situación quedó claramente de manifiesto tras el retiro de la subvención con que el Estado ayudaba al financiamiento del Asilo, lo que en la práctica terminaría obligando a determinar su cierre en febrero de 1885. El Presidente de la República, Domingo Santa María, justificó su decisión señalando que ahora sería el Estado el que se haría cargo del cuidado y la educación de los huérfanos de la guerra. Para dar cuenta de tal propósito se establecerían escuelas prácticas de agricultura y minería, en Copiapó, Coquimbo, Santiago y Concepción, en las que los menores recibirían instrucción con cargo al erario nacional y sin intermediarios de la Iglesia Católica. De forma evidente, el conflicto laico-clerical, que marcó con su impronta muchas de las disputas políticas del siglo XIX y que tendrían un hito relevante en la aprobación de las llamadas leyes laicas en 1883 y 1884, hizo sentir sus efectos en la percepción y valoración que se tenía del hogar de los huérfanos de la guerra.

En agosto de 1885 el Gobierno inauguró con gran pompa la Escuela Práctica de Agricultura de Santiago, sin embargo, esta institución estuvo lejos de cumplir con los objetivos con que se había creado, pues para llenar sus vacantes fue necesario recurrir a huérfanos de la Casa de la Providencia e incluso a algunos niños araucanos, traídos del sur especialmente para este propósito. Para nuestro caso, lo relevante de la decisión de Santa María y del cierre del Asilo de la Patria radica en que al establecer las escuelas prácticas el Estado por primera vez asumía de manera directa la responsabilidad por el cuidado de los menores en condición de orfandad. Si bien en su sentido original tal interés surgió por la condición especial de aquellos menores, al ser "huérfanos de la guerra", dicha decisión fue una clara señal de un evidente cambio en las políticas estatales en relación con la orfandad como problema social.

Bajo el mismo gobierno de Santa María también fue relevante la inauguración del nuevo edificio de la Casa de Huérfanos de Santiago en 1884, con lo que se concretó un anhelo largamente esperado y que había sufrido numerosas postergaciones desde la década de 1850, motivadas principalmente por las estrecheces económicas que afectaban al Estado. La habilitación de las nuevas instalaciones representó, sin duda, un hito en la trayectoria de esta institución y fue reflejo, asimismo, del interés con que el Estado comenzaba a ponderar la importancia de la beneficencia pública y, en términos específicos, una muestra de la toma de conciencia respecto a la urgencia con que debían ser satisfechos los requerimientos de los huérfanos. A diferencia del Asilo de la Patria, la administración del establecimiento siguió siendo responsabilidad de religiosas, en este caso, de la Congregación de las Hermanas de la Providencia, quienes estaban a su cargo desde 1853 y continuarían trabajando de forma coordinada con el 
Estado hasta mediados del siglo XX (Milanich 2009).

Esta mayor incumbencia del Estado en materia de beneficencia pública también se vio reflejada en sucesivos ajustes a la legislación que la regulaba. Tras ańos de discusiones se aprobó en enero de 1886 un nuevo Reglamento Orgánico de las Juntas de Beneficencia que consolidó a estas organizaciones como responsables de esta materia. Las ahora llamadas Juntas de Beneficencia Departamentales quedarían a cargo de las diversas instituciones que tradicionalmente habían formado parte de su esfera de influencia, como hospitales, hospicios, lazaretos, dispenserías de medicamentos, hospicios de insanos, asilos de expósitos y huérfanos y cementerios, tomando también bajo su control a toda institución que recibiera recursos públicos y estatales (Ponce de León 2011). Al año siguiente se promulgaron nuevas leyes con las que se constituyó la Junta Central de Salubridad, que tenía por finalidad asesorar al gobierno en materias vinculadas con la salubridad pública pudiendo crear dispensarias, lazaretos, trasladar enfermos e inhumar cadáveres. Esta organización representó un antecedente directo del Consejo Superior de Higiene Pública, que sería creado en 1892. Los continuos traspiés provocados por sucesivas y mortíferas epidemias obligaron a replantearse el rol que se tenía la concepción tradicional de la beneficencia. Poco a poco la ciencia médica se impondría como la alternativa viable para la resolución de estos problemas, sin embargo, no será sino hasta el siglo XX cuando el Estado asuma un rol marcadamente asistencial en estas materias dándole a los médicos la responsabilidad principal de su funcionamiento.

\section{CONCLUSIONES}

Tras la Independencia uno de los desafíos más relevantes enfrentado por las emergentes naciones latinoamericanas fue la creación de un aparato estatal que permitiera la administración y garantizara la pervivencia de estas nuevas entidades en el tiempo. Debido a ello, durante el siglo XIX el proceso de construcción del estado-nación fue complejo y experimentó obstáculos de diversa índole. En primer lugar, no existía un claro consenso en torno a la forma en que se debían organizar y conducir políticamente, careciendo además de una burocracia capacitada que administrara la institucionalidad que se estaba creando. Junto con estos factores, las dificultades económicas hicieron que este proceso fuera más complejo, pues las limitaciones financieras entorpecían el emprendimiento de cualquier proyecto de mediano o largo plazo. Dado este contexto, resulta razonable entender por qué las problemáticas sociales que afectaban a los habitantes de estas naciones recibieron poca atención por parte de las autoridades políticas. 
A lo largo del siglo XIX la beneficencia pública fue mirada con poco interés por parte del Estado chileno debido a dos motivos principales. En

primer lugar, se consideraba que dicha tarea debía ser dejada a la voluntad de filántropos y/o religiosos que se sintiesen llamados a colaborar en tal tarea. En segundo término, las estrecheces económicas que continuamente experimentaba el erario fiscal obligaron a priorizar la inversión estatal en otras materias dejando de lado iniciativas en esta área. Dada esta situación, fueron los particulares quienes se organizaron, en la forma de asociaciones y sociedades de beneficencia para colaborar con aquellos desfavorecidos que lo requerían: la situación de los huérfanos fue objeto de especial atención en este contexto dado el impacto social que generaba este fenómeno. En su tarea fueron tradicionalmente auxiliados por eclesiásticos y religiosas quienes interesados en mantener su posición en la esfera pública y, al mismo tiempo, genuinamente alarmados por los dramas humanos que con frecuencia presenciaban, pusieron todos los recursos de la Iglesia Católica en favor de la resolución o al menos el aplacamiento de estas problemáticas sociales.

En las últimas décadas del siglo XIX este panorama cambiará de manera importante. Por un lado, el Estado, ya más consolidado institucionalmente y con una clara impronta liberal, tomará a su cargo de forma inédita algunas de las iniciativas de auxilio a los huérfanos más relevantes del periodo profundizando con ello su disputa con la Iglesia Católica. El caso del Asilo de la Patria es plenamente representativo de esta situación. Por otro lado, un Estado con mayores recursos, situación consolidada tras la Guerra del Pacífico, pudo emprender nuevas iniciativas, ya en la forma de infraestructura, como lo fue la inauguración del edificio de la nueva Casa de Huérfanos de Santiago, o bien en materia de reorganización institucional, demostrada en la creación de nuevas agencias y oficinas estatales que tuvieron como ámbito de acción la beneficencia pública. La atención por parte del Estado de la beneficencia pública, en general, y de los huérfanos, en particular, refleja en buena medida el desarrollo y la evolución del Estado chileno durante el siglo XIX. 


\section{REFERENCIAS}

Cárdenas, M. (1991). Grupos Marginados en los Inicios de la Era Republicana: Vagabundos, Mendigos e Indigentes. Cuadernos de Historia, 11, 47-61.

Delgado, M. (1984). Marginación e Integración Social en Chile Colonial: Los Expósitos 1770-1830. Tesis (Licenciatura en Historia), Universidad Católica de Valparaíso, Chile.

Durán, B. (1851). Elogio Fúnebre del Señor Don José Miguel de la Barra. Que pronunció en las Exequias Solemnes Celebradas en la Iglesia del Asilo del Salvador, el Presbitero don Buenaventura Durán, el Día 19 de Mayo de 1851. Santiago: Imprenta de la Sociedad.

Gatica, J. M. (1884). Asilo de la Purísima. Primera Asamblea Jeneral de la Unión Católica de Chile. Celebrada en Santiago en 1', 2, 4 i 6 de Noviembre de 1884. Santiago: Imprenta Victoria.

Home, D. (2007). Los Huérfanos de la Guerra del Pacífico: El Asilo de la Patria', 1879-1885. Santiago: LOM, DIBAM

La Revista Católica. (1844). Asilo del Salvador. La Revista Católica, 45, 1 de noviembre.

Lamarca, P. (1859). Memoria que la Presidenta de la Sociedad del Asilo del Salvador Presenta a sus Consocias. Reunidas en Junta Jeneral al Terminarse el Año de su Administración, con Arreglo al Título IV Artículo II de sus Estatutos. Valparaíso: Imprenta y Librería del Mercurio.

Laval, E. (1949). Origen de las Juntas de Beneficencia. Revista MédicoAsistencial, 1, 43-47.

Milanich, N. (2009). Children of fate: Childhood, Class and the State in Chile, 1850-1930. Durham: Duke University Press.

Montecino, S. (1991). Madres y Huachos. Alegorias del Mestizaje Chileno. Santiago: Cuarto Propio.

Pinto, J. y Salazar, G. (2002). Historia Contemporánea de Chile V. Niñez y Juventud. Santiago: LOM.

Ponce de León, M. (2011). Gobernar la Pobreza. Prácticas de Caridad y Beneficencia en la Ciudad de Santiago, 1830-1890. Santiago: DIBAM, Editorial Universitaria. 
Home

Riveros, M. (1849). Proyecto sobre la Erección de una Casa de Huérfanos en esta Ciudad de La Serena, Capital de Provincia de Coquimbo. Discurso Pronunciado ante la Sociedad de Beneficencia, en su Sesión General del día 17 de Setiembre, por uno de sus Miembros. Valparaíso: Imprenta Europea.

Román, M. (1886). Vida del Señor Presbitero Don Blas Cañas Fundador de la Casa de María y del Patrocinio de San José. Santiago: Imprenta Católica de Manuel Infante.

Román, M. (1887). La Casa de María. Relato Histórico Presentado a la Asamblea Católica de 1886. Santiago: Imprenta de La Unión.

Salazar, G. (1990). Ser Niño "Huacho" en la Historia de Chile (Siglo XIX). Proposiciones, 19, 55-83.

Saldivia, Z. y G. de la Jara. (2001). La Sociedad Nacional de Agricultura en el Siglo XIX Chileno: Su Rol Social y su Aporte al Desarrollo Científico-Tecnológico. Scripta Nova. Revista Electrónica de Geografía y Ciencias Sociales, V (100).

Salinas, R. (1983). Salud, Ideología y Desarrollo Social en Chile: 18301950. Cuadernos de Historia, 5, 99-126.

Sunkel, O. (2011). El Presente como Historia: Dos Siglos de Cambio y Frustración en Chile. Santiago: Catalonia.

Vicuña Mackenna, B. (1974). Médicos de Antaño. Buenos Aires: Editorial Francisco de Aguirre.

Recibido: 15-05-2015

Aceptación de la versión final: 19-06-2015 